\title{
La diffusion de la
} révision hiéronymienne des traductions bibliques dans les latins (ve-XIle siècle): l'exemple des Douze Prophetes

\section{Marie Frey Rébeillé-Borgella*}

Les études sur l'histoire des révisions hiéronymiennes des traductions latines de la Bible, ${ }^{1}$ leur réception et leur diffusion dans l'Antiquité tardive et au Moyen Âge portent principalement sur les manuscrits (rassemblés ou non dans des Bibles unifiées) et fragments codicologiques connus, ainsi que sur l'utilisation de la révision hiéronymienne chez les auteurs chrétiens et dans les lectionnaires latins. ${ }^{2}$ D'autres sources restent encore à explorer. Parmi elles, le corpus liturgique représent par

* Laboratoire hisoma (Histoire et Sources des Mondes Antiques) - Umr 5189, Sources Chrétiennes, 22, rue Sala -69002 Lyon ; marie.rebeille-borgella@ orange.fr.

1 Comme les révisions des traductions latines effectuées par Jérôme de Stridon n'ont pas, au début de leur diffusion, circulé sous forme de Bibles réunissant tous les livres du canon, il nous paraît peu opportun, pour la période considérée, de parler de Vulgate et de diffusion de la Vulgate. C'est pourquoi, autant que faire se peut, nous préférons parler de révisions hiéronymiennes des traductions bibliques.

2 Voir, entre autres, Berger, Histoire de la Vulgate pendant les premiers siècles du Moyen Âge; Quentin, Mémoire sur l'établissement du texte de la Vulgate; Metzger, Early Versions of the New Testament; Salmon, «Le texte biblique des lectionnaires mérovingiens". 
les sacramentaires. En effet, depuis ceux de Carl Marbach, ${ }^{3}$ rares sont les travaux qui ont étudié les liens entre Bible et liturgie sous l'angle des versions bibliques utilisées dans les sacramentaires. La présente communication s'intéresse ainsi à l'intégration du texte hiéronymien dans les sources liturgiques de l'Antiquité tardive et à la survie des versions vieilles latines dans la liturgie occidentale. Elle étudie quelques cas de mention et citation des livres dits des Douze Prophètes ${ }^{4}$ jusqu'au XIIe siècle. Le choix de cette période chronologique a été dicté par une volonté de prise en compte des livres liturgiques utilisés dans la péninsule ibérique pendant la domination musulmane. ${ }^{5}$

Avant d'entrer dans l'examen des relevés, une question se pose : quels sont les types de traces de versets bibliques qui permettent de différencier un texte de type vieux-latin du texte révisé par Jérôme ? On distingue habituellement trois manières d'insérer une œuvre extérieure dans un texte : l'allusion, la mention et la citation. L'allusion consiste à évoquer le contenu du texte sans le citer mais en employant des termes qui vont immédiatement renvoyer l'auditeur au référent commun qu'est le texte biblique dont il est question. La mention peut consister dans le réemploi de quelques mots du texte extérieur. Nous considérons qu'il s'agit d'une mention et non d'une allusion dès que le texte liturgique cite deux mots dont l'association est caractéristique d'un verset identifiable d'un livre biblique. La citation se caractérise par un réemploi plus long, d'un segment de phrase, d'une phrase intégrale ou de plusieurs phrases. Pour étudier la diffusion de la Vulgate et l'éventuelle persistance des Vieilles Latines dans les textes liturgiques, il nous faut donc nous concentrer sur les mentions et les citations, c'est-à-dire les cas où le texte biblique est repris tel quel et inséré dans le tissu du texte liturgique. Le corpus obtenu est maigre. Les exemples que nous avons retenus posent néanmoins plusieurs problèmes textuels qui interrogent sur le rapport des rédacteurs de ces livres liturgiques aux différentes familles de traductions latines ayant circulé.

Carmina Scripturarum scilicet Antiphonas et Responsoria ex sacro Scripturae fonte in libros liturgicos Sanctae Ecclesiae Romanae derivata.

4 Nous avons centré notre étude sur les mentions et citations insérant une portion de texte biblique ne pouvant venir que des livres des Douze Prophètes. Les mentions du catulus leonis (syntagme attesté quatorze fois dans la traduction de l'Ancien Testament révisé par Jérôme) ou des triticum, uinum et oleum (cités dans le Deutéronome avant de l'être dans les livres d'Osée, de Joël et d'Aggée) ont ainsi été exclues de notre étude.

5 Les corpus liturgiques dépouillés pour cette étude sont le Corpus orationum, CCSL 160-160m (désormais Co), le Corpus praefationum, CCSL 161-161D (désormais $C P$ ), le Corpus benedictionum pontificalium, CCSL 162-162C (désormais CBP) et le Missale Gothicum e codice Vaticano Reginensi latino 317 editum, CCSL 159D. 


\section{LE TEXTE VIEUX-LATIN ET LE TEXTE DE LA VULGATE SONT IDENTIQUES}

Le premier cas rencontré ${ }^{6}$ est celui des mentions ou citations des livres des Douze Prophètes pour lesquelles il n'est pas possible de déterminer si la traduction latine employée par les rédacteurs du texte liturgique est la révision de Jérôme ou une version vieille latine, les deux comportant la même traduction du texte concerné.

La prière des prêtres d'Israël citée dans le livre de Joël $1(2,17)$, parce Domine populo tuo $(\mathrm{Vg})$ est reprise dans plusieurs prières liturgiques chrétiennes. Le texte est celui de la révision hiéronymienne. La consultation de la base de données BiblIndex nous permet de vérifier que le verset n'est pas attesté chez les Pères latins avant le Commentaire sur les petits prophètes de Jérôme qui le cite. ${ }^{7}$ Nous savons néanmoins que Jérôme a repris ici un texte vieux-latin grâce au manuscrit Paris BN 13409 qui transmet des fragments vieux-latins des petits prophètes, dont $\mathrm{Jl} 2,25 .{ }^{8}$ Une oraison qui se trouve dans les sacramentaires léonien, grégorien, dans les sacramentaires gélasiens d'Angoulême et de Gellone, gélasien mixte de Monza, de Saint-Gall et de Rheinau, reprend les quatre termes:

Parce, Domine, parce populo tuo, ut, dignis flagellationibus castigatus, in tua miseratione respiret. $(\mathrm{co}, 4130)$

Épargne, Seigneur, épargne ton peuple, afin que, purifié par des pénitences mérités, il revive en ta pitié.

De même, une prière du missel de Bobbio cite la formule entière :

Deus [...] parce, Domine, parce populo tuo, ne tradas bestiis animas confitentes tibi. $(\mathrm{co}, 1276)$

Épargne, Seigneur, épargne ton peuple, ne livre pas aux bêtes les âmes qui te louent.

D'autres oraisons n'empruntent au verset biblique que les deux premiers mots, Parce Domine. La référence au livre de Joël ne fait

6 Tous les relevés cités dans cet article ont été effectués grâce à la base de données The Library of Latin Texts.

7 Commentarii in prophetas minores: In Ioelem, CCsL 76, 2.290, 298, 342, 379, 425.

8 Frede et al., « Reste einer Prophetenhandschrift ». 
pour autant aucun doute, celui-ci étant le seul à attester la formule dans les Bibles latines. Parmi ces oraisons, une remonte au Sacramentaire Léonien et est commune aux liturgies grégorienne, gélasienne et ambrosienne :

Parce, Domine, parce peccantibus et, ut ad propitiationem tuam possimus accedere, spiritum nobis tribue corrigendi. (co, 4128)

Épargne, Seigneur, épargne les pécheurs et, afin que nous puissions nous approcher de ta miséricorde, accorde-nous l'esprit de correction.

Les oraisons 4129a et 4129b, de formulations très proches, commencent par la formule "parce, Domine, parce peccatis nostris » (4129a). Enfin, loraison 4931 reprend elle aussi la formule parce Domine + datif: parce, domine, parce supplicibus [...]

\section{LA TRADUCTION UTILISEE PAR LES TEXTES LITURGIQUES EST IDENTIFIABLE SANS AMBIGUÏTE}

\subsection{L'emploi des Vieilles Latines}

Notre corpus comprend des cas de recours aux Vieilles Latines sans qu'aucune ambiguité sur la version utilisée ne soit possible. C’est le cas de la préface pour la messe du jour de Pâques $\left(\mathrm{n}^{\circ} 280\right)^{9}$ du Missale Gothicum :

Hic enim Dominus noster Iesus Christus filius tuus adimplens prophetias temporibus praestitutis uisitauit nos post biduum, die tertia resurrexit.

En effet, ici, notre Seigneur, ton fils Jésus Christ, accomplissant les prophéties aux temps fixés par avance, nous a visité après deux jours et est ressuscité le troisième jour.

La prière cite Os. 6,3, dont la traduction hiéronymienne est la suivante:

uiuificabit nos post duos dies, in die tertia suscitabit nos ${ }^{10}$

9 Missale Gothicum, éd. par Els Rose.

10 Le texte biblique que nous citons en regard des pièces liturgiques est systématiquement le texte hiéronymien, afin de permettre la comparaison entre la version du texte liturgique et celle de Jérôme. 
Il nous ramènera à la vie dans deux jours, il nous ressuscitera le troisième jour.

L'emploi de post biduum par le Missale Gothicum est un marqueur lexicologique caractérisant un texte vieux-latin, puisqu'on ne le trouve dans aucun manuscrit de la Vulgate d'Osée. Il existe cependant d'autres variantes vieilles-latines de ce syntagme : le verbe sanare, ${ }^{11}$ et non uiuificare, est attesté chez Tertullien, Rufin, Augustin et Quodvultdeus. Le uisitare du Missale Gothicum est pour sa part attesté dans deux autres textes tardo-antiques : le Sermo de symbolo (CPL 1759), d'origine hispanique et datant, selon Liuwe H. Westra, du ve siècle, ${ }^{12}$ et un Commentaire sur Marc attribué à Jérôme mais composé en réalité au vire siècle, qui pourrait être d'origine irlandaise. ${ }^{13}$

Cette attestation d'une trace des Vieilles latines dans cette préface ne permet pour autant pas d'établir une généralité sur les traductions bibliques employées par le Missale Gothicum, et ce d'autant plus que les autres attestations des livres des petits prophètes dans ce missel sont des versets dont les leçons vieilles latines et les leçons de la Vulgate sont identiques.

\subsection{L'emploi de la traduction hiéronymienne}

Un autre cas est celui où l'identification du texte hiéronymien comme source pour la rédaction du texte liturgique ne fait pas de doute. C'est le cas de l'emploi d'Amos 6, 5 :

qui canitis ad uocem psalterii sicut Dauid

vous qui chantez au son de la cithare comme David

Ce verset est utilisé dans le syntagme liturgique beatus Dauid rex in psalterio, présent dans une bénédiction des sacramentaires gélasiens d'Angoulême, Autun et Gellone ainsi que d'un sacramentaire de

11 Tertullien, Aduersus Marcionem 4; Rufin, Expositio symboli 28 ; Augustin, De ciuitate Dei 18.28 ; Quoduultdeus, Liber promissionum et praedictorum Dei 3.29.

12 Westra, The Apostle's Creed, 371-378.

13 Cf. Expositio Euangelii secundum Marcum, ccsL 82. Marc Cahill explique que son travail « n'a pas été à même de confirmer l'hypothèse [d'une origine irlandaise du commentaire] de Bischoff, mais ne l'a pas non plus clairement invalidée ». 
Reims du xe siècle. Il est le seul verset de la révision hiéronymienne à montrer David jouant d'un psalterium. ${ }^{14}$

Vi<si>ta eum. sicut Moysen. in rub[r]o. Iesuae. in agro. Iesu Naue. in proelio. Samuelem. crinitum in templo. et illa eum. promissione. sideria $[\mathrm{m}]$ ac sapientie tue. rore perfunde. quia beatus Dauid rex in psalterio Solomon[em] filius eius te remunerante. percipit e caelo. ${ }^{15}$

Visite-le comme Moïse dans le buisson ardent, comme Josué dans le champ, comme Josué fils de Nun dans le combat, comme le chevelu Samuel dans le temple, et inonde-le selon cette promesse de la rosée céleste et de ta sagesse, parce que le bienheureux roi David sur sa cithare, fils de Salomon, l'a reçue du ciel alors que tu la lui accordais.

(Sacramentaire gélasien d'Autun)

Visita eum interuentu illius sicut Moysen in rubo, Iosue in agro, Iesu Naue in prelio, Samuhel crinitum in templo. Et illa eum promissione siderea hac sapientiae tuae rore perfunde, qua beatus Dauid rex in psalterio, Salomon filius eius te remunerante percepit e caelo.

Visite-le par son intercession comme Moïse dans le buisson ardent, comme Josué dans le champ, comme Josué fils de Nun dans le combat, comme le chevelu Samuel dans le temple. Et inonde-le selon cette promesse céleste de la rosée de ta sagesse, que le bienheureux roi David sur sa cithare, fils de Salomon, a reçue du ciel alors que tu la lui accordais.

(Sacramentaire d'Angoulême, 1858) ${ }^{16}$

Visita eum sicut Moysen in rubo, Iosue in agro, Iesu Naue in praelio, Samuelem crinitum in templo; et illa eum promissione siderea ac sapientiae tuae rore perfunde, quo beatus Dauid rex in psalterio, Salomon filius eius te remunerante percepit e caelo.

142 Chr 29, 25 associe aussi les termes latins Dauid et psalterium. Le récit du chapitre 25 montre le roi Ezékias ordonnant un holocauste selon les commandements de David, ceux-ci incluant une prière au son des instruments. Ce n'est donc pas la figure de David jouant de la cithare qui est mise en scène dans ce verset. Am 6, 5 est donc la seule référence biblique des prières mentionnées ici.

15 La ponctuation que nous reproduisons est toujours celle des éditions consultées, ici Liber Sacramentorum Augustodunensis, CCSL 159B. Liber Sacramentorum Engolismensis, CCSL 159C. 
Visite-le comme Moïse dans le buisson ardent, comme Josué dans le champ, comme Josué fils de Nun dans le combat, comme le chevelu Samuel dans le temple. Et inonde-le de cette promesse céleste et de la rosée de ta sagesse, comme le bienheureux roi David sur la cithare, fils de Salomon, l'a reçue du ciel alors que tu la lui accordais.

(Sacramentaire d'Angoulême, 2318) ${ }^{17}$

Visita eum interuentu sanctorum omnium sicut Moysen in rubo, Iosuae in agro, Iesu naue in proelio, Samuel meruit crinitus in templo. Et illa aeum permissione siderea ac sapientiae tuae rore perfunde qua beatus Dauid rex in psalterio psalmorum filius <eius> te remunerante percepit a caelo.

Visite-le par l'intercession de tous les saints comme Moïse dans le buisson ardent, comme Josué dans le champ, comme Josué fils de Nun dans le combat, comme le chevelu Samuel dans le temple l'ont mérité. Et inonde-le éternellement de cette promesse céleste de la rosée de ta sagesse, que le bienheureux roi David sur la cithare des Psaumes, son fils, a reçue du ciel alors que tu la lui accordais.

$(\text { Sacramentaire de Gellone, 2092 })^{18}$

Vocem psalterii est un syntagme davantage hiéronymien que vieux-

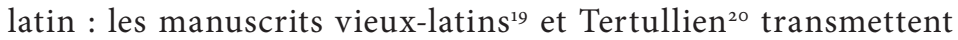
organorum ou organi au lieu de psalterii. Il y a donc tout lieu de penser que la rédaction des oraisons citées s'appuie sur le terme hiéronymien et non sur celui vieux-latin.

De même, une prière pour la paix des litaniae canonicae du rituel hispanique de l'abbaye de Silos (xe-xie siècle) utilise en réemploi la traduction de Jérôme de $\mathrm{Jl}$ 2, 13 :

Scindentes corda nostra et non vestimenta, tuam, Deus summe, imploramus clementiam, ut [...] (co, 56462).

En déchirant nos cœurs et non nos vêtements, Dieu très-haut, nous implorons ta clémence, afin que [...]

17 Ibid.

18 Liber Sacramentorum Gellonesis, CCsL 159.

19 Ms 175, cf. Gryson, Altlateinische Handschriften, 267-269.

20 Adversus Marcionem, CCSL 1, 4.15. 
et scindite corda vestra et non vestimenta vestra et convertimini ad Dominum Deum vestrum

Et déchirez vos cœurs et non vos vêtements, et revenez vers le Seigneur votre Dieu (J1 2, 13)

Scindite est une traduction hiéronymienne. En effet, parmi les témoins du texte vieux-latin, le manuscrit Paris Bibliothèque Nationale Lat. $13409^{21}$ contient disrumpite et le texte africain cyprianique ${ }^{22}$ donne discindite.

\section{LES CITATIONS PROBLEMATIQUES : REVISION HIERONYMIENNE OU TEXTE VIEUX-LATIN?}

\subsection{Malachie 3, 1}

Dans le cas d'un verset de l'Ancien Testament repris dans le Nouveau Testament, quel est le livre biblique qu'avaient le plus probablement en tête les rédacteurs de la prière ? Plusieurs lectionnaires montrent que les péricopes de Malachie et des évangiles sont associées lors du temps de l'Avent. Ainsi, deux des textes de l'office du ze dimanche de l'Avent indiqués dans le Liber Comicus, lectionnaire hispanique du xire siècle, sont la péricope de Malachie $(3,1-4)$ et le début de l'Évangile de Marc $(1,1-8)$.

Une mention de ce verset se trouve dans l'oraison du temps de l'Avent 2553 :

Excita, Domine, quaesumus, corda nostra ad praeparandas unigeniti tui vias, ut per eius adventum purificatis tibi mentibus servire mereamur.

Incite nos cœurs, Seigneur, nous t'en prions, à préparer les chemins de ton fils unique, afin que nous méritions de t'adorer dans nos esprits purifiés par ta venue.

Or Malachie 3, 1 est cité par les évangiles de Matthieu $(11,10)$ et Luc $(7,27)$ : 
Ecceego mittamangelum meumet praeparabit uiam antefaciem meam. $\left(\mathrm{Ml}_{3,1}\right)$

Voici que, moi, j'enverrai mon messager et qu'il préparera le chemin devant ma face.

Hic enim est de quo scriptum est ecce ego mitto angelum meum ante faciem tuam qui praeparabit viam tuam ante te (Mt 11, 10)

C'est en effet de lui qu'il a été écrit : Voici que j'envoie mon messager devant ta face, lui qui préparera le chemin devant toi.

Sicut scriptum est in Esaia propheta ${ }^{23}$ ecce mitto angelum meum ante faciem tuam qui praeparabit viam tuam $(\mathrm{Mc} 1,2)$

Ainsi qu'il est écrit dans le prophète Isaïe : Voici que j'envoie mon messager devant ta face, lui qui préparera ton chemin.

Cette oraison est présente dans le Sacramentaire gélasien ancien (du viııe siècle). Elle est attestée à la même époque dans le Sacramentaire gélasien grégorianisé conservé à Prague, dans les Missels de Bobbio et le Missel Gallican. Sa formulation est reprise employée comme collecte et reprise dans une préface du temps de l'Avent dans le missel gallican du viIIe siècle. Quel texte cette pièce cite-t-elle précisément ? Là où l'oraison utilise un pluriel alors que la Vulgate a recours au singulier, la tradition manuscrite de la traduction hiéronymienne de Mt 11, 10 et de Lc 1, 2 donne la même lecture que les manuscrits vieux-latins de ces deux évangiles : viam y est au singulier, la variante vias, celle de l'oraison, n'est pas attestée. Mais, en ce qui concerne Malachie, si nous pouvons nous appuyer sur des manuscrits transmettant le texte de la Vulgate, les fragments donnant des péricopes vieilles-latines de ce livre ne contiennent pas ce verset. Il nous faut chercher ailleurs des traces du texte vieux-latin. Le verset est cité intégralement sous la forme employée par notre oraison dans la traduction de Rufin des Homélies sur Josué24 d'Origène :

Exploratores isti, qui mittuntur 'ante faciem' Iesu, possunt et angeli Dei putari, sicut scriptum est : ecce mitto angelum meum ante faciem tuam, qui praeparabit vias tuas.

23 L'évangéliste attribue faussement le verset à Isaïe : celui-ci est bien du livre de Malachie.

24 Origène, Homélies sur Josué 3.3. 
Ces chercheurs, qui sont envoyés 'devant la face' de Jésus, peuvent aussi être considérés comme des messagers de Dieu, ainsi qu'il est écrit : voici que j'envoie mon messager devant ta face, lui qui préparera tes chemins.

Raban Maur, au Ixe siècle, a recours à la même version dans son Commentaire sur Josué, ${ }^{25}$ qui cite mot pour mot les Homélies sur Josué :

Exploratores isti qui mittuntur ante faciem Iesu possunt et angeli Dei putari, sicut scriptum est: 'Ecce mitto angelum meum ante faciem tuam, qui praeparat vias tuas ante te'.

Les autres attestations patristiques de praeparare vias sont des mentions ou citations de Lc 1,76 :

et tu puer propheta Altissimi vocaberis praeibis enim ante faciem Domini parare vias eius

Et toi, petit enfant, tu seras appelé prophète du Très-Haut. En effet, tu prépareras ses chemins devant la face du Seigneur.

Tous les autres pères (Tertullien, Ambroise, Gaudence de Brescia, Chromace d'Aquilée, Maxime de Turin, Augustin) qui citent le verset de Malachie ou des évangiles le citent avec viam. La formulation de l'oraison 2553 ne reflète donc en aucun cas une tradition textuelle solide. Soit elle est dépendante de la traduction d'Origène par Rufin, soit elle puise, mais indépendamment, aux mêmes sources que Rufin (un texte vieux-latin perdu ?), soit il s'agit d'une mention libre du rédacteur du Missel. Il n'est pas possible de trancher.

\subsection{Joël 2, 11}

Une autre prière de notre corpus comprend une mention biblique problématique. Il s'agit d'une oraison post pridie ${ }^{26}$ de la messe de l'Ascension attestée dans des livres hispaniques pré-grégoriens pour la messe de l'Ascension : gallicanes. 
Quod scientes, Domine, ut nobis dies illa terribilis aliquantulum ex tua propitiatione mitior adveniat, haec munera offerimus.

Parce que nous savons, Seigneur, que ce jour terrible adviendra un tant soit peu adouci par ta miséricorde, nous t’offrons ces présents.

Elle fait mention d'un verset du livre de Joël $(2,11)$, qui est la seule attestation du syntagme dies terribilis dans l'ensemble des traductions révisées par Jérôme :

magnus enim dies Domini et terribilis ualde

S'agit-il d'un texte vieux-latin ou du texte de la Vulgate ? L'oraison est uniquement hispanique : elle est attestée dans six manuscrits de la liturgie wisigothique provenant de l'abbaye de Silos de Tolède. Parmi ces manuscrits, seul le Liber misticus est, selon la datation donnée par Anscari Mundó, ${ }^{27}$ antérieur à la conquête de Tolède en 1085. La question se pose de savoir si ce livre liturgique est tributaire de témoins bibliques vieux-latins. L'oraison qui nous concerne ne peut avoir comme source biblique que le livre de Joël, qui est le seul des livres hébreux révisé par Jérôme à attester le syntagme dies terribilis. La grande majorité des témoins vieux-latins européens dont nous avons connaissance n'emploient pas terribilis dans ce verset. Or dies terribilis est aussi attesté dans un Commentaire sur Job anonyme d'origine arienne, ${ }^{28}$ dont Kenneth Steinhauser ${ }^{29}$ date la rédaction entre 384 et 387. La majorité des études sur la datation des révisions de Jérôme estime que celle des livres des Douze Prophètes a eu lieu entre 390 et $3944^{30} \mathrm{Si}$ on suit cette hypothèse et qu'on admet dans le même temps celle de Kenneth Steinhauser, alors dies terribilis est aussi une variante vieille-latine. Mais nous ne pouvons pas en avoir la certitude absolue. Les lectures des litaniae minore ${ }^{31}$ du Liber Comicus de Tolède, un lectionnaire hispanique du milieu du xire siècle dont le texte du Nouveau Testament est vieux-latin,,$^{32}$ comprennent la péricope des deux premiers chapitres du livre de Joël : celles-ci ont comme texte

Mundó, «La datación de los códices liturgicos visigóticos toledanos », 1-25.

Anonymus in Iob commentarius, CSEL 96.

Steinhauser, « Job in Patristic Commentaries and Theological Works », 36.

Jérôme, Préfaces aux livres de la Bible, éd. par A. Canellis, 98.

Liber Comicus, sive lectionarius missae quo Toletana ecclesia ante annos mille et ducentos utebatur, Analecta Maredsolana 1.

Metzger, Early Versions, 304. 
pour Jl 2, 11 dies terribilis. Les rédacteurs du Liber Comicus se sont-ils appuyés sur des traductions différentes de la Bible pour l'Ancien et le Nouveau Testament ou bien ont-ils cité le livre de Joël dans sa version vieille-latine, à laquelle Jérôme n’aurait pas touché pour le syntagme qui nous intéresse? Aucun élément n’est décisif.

Un autre emploi liturgique de dies terribilis se trouve dans une préface pour les messes dominicales du Missel de Bobbio, qui cite Jl 2, 11 puis So 1, 15-16 (dies irae, dies tribulationis...) :

O quam gravis et laboriosa es $<t>$ dies illa! O quam terribilis et horribilis es $<t>$ dies illa, dies irae, dies tribulationis et angustiae, dies calamitatis et miseriae, dies tenebrarum et caliginis, dies nebulae et turbidinis, dies tubae et clangoris (Corpus praefationum, 149o)

Ô quel jour pénible et misérable est ce jour ! Ô quel jour terrible et horrible est ce jour, jour de colère, jour de tourment et d'angoisse, jour de détresse et de misère, jour de ténèbres et de brouillard, jour d'obscurité et de tempête, jour de trompette et de grands cris

Le texte de Sophonie cité est celui révisé par Jérôme. Cependant, la même préface, 1490, utilise une citation d'un verset qui n'est probablement pas issu de lentreprise de traduction hiéronymienne :

Ille venturus manus tenet ad iudicandum, ubi clavi confixae sunt, et ips a signa permane $<n>t$ in aeternum, sicut scriptum est : 'videbunt $<$ in $>$ quem transfixerunt', et : 'plangent se cunctae tribus terrae'.

Cette main qui vient retient pour juger, là où les clous sont fixés, et ces signes demeurent pour l'éternité, ainsi qu'il est écrit : 'ils verront celui qu' ils ont transpercé', et 'toutes les tribus de la terre se lamentent'.

Il s'agit d'un verset de l'Apocalypse dont la version ne provient probablement pas des révisions opérées par Jérôme ou ses continuateurs. Mais aucun manuscrit, ni vieux-latin ni hiéronymien, ne transmet la leçon cunctae tribus. Le Missel de Bobbio dépend-il pour cette pièce d'une traduction perdue? Quoi qu'il en soit, il semble que cette pièce ait recours, pour une même oraison, à plusieurs traditions de traductions bibliques, ce qui laisse ouverte la possibilité que dies terribilis, en $\mathrm{Jl} 2,11$, soit également une traduction vieille-latine minoritaire. Une seule et même pièce mélange-t-elle différentes traductions latines ? Rien ne permet de le dire avec certitude. 


\section{LES CAS OÙ LES VIEILLES LATINES DEVIENNENT LA TRADITION}

\subsection{Jonas 2, 1}

Le corpus étudié comprend enfin deux cas d'usage persistant dans la tradition liturgique des Vieilles Latines, concomitamment ou non avec l'usage de la traduction hiéronymienne.

Le premier concerne la mention de Jonas 2, 1 :

et erat Iona in uentre piscis tribus diebus et tribus noctibus

Et Jonas était dans le ventre du poisson pendant trois jours et trois nuits.

Les Vieilles Latines, traduisant sur la Septante - qui emploie le terme

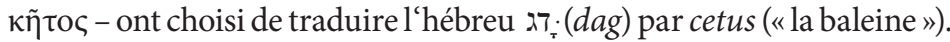
Dans les textes liturgiques latins faisant mention du séjour de Jonas dans le ventre de la baleine, le syntagme vieux-latin ventris ceti (deux occurrences dans notre corpus) et le syntagme hiéronymien ventris piscis (deux occurrences) sont tous deux attestés. Mais ventris ceti est bien plus employé que ventris piscis chez les auteurs tardo-antiques et médiévaux (plus d'une centaine d'occurrences pour le premier, une trentaine pour le second). Les deux emplois de ventris ceti dans le corpus liturgique sont les suivants :

Exaudi me, Deus, te de totis praecordiis invocantem, qui exaudisti Ionam de ventre ceti orantem. (Corpus praefationum, 445, Pontifical du Liber Ordinum de Silos)

Exauce-moi, ô Dieu, moi qui t'invoque du fond de toutes mes entrailles, toi qui as exaucé Jonas quand il priait du fond du ventre de la baleine.

Libera, Domine, anima $<m>$ famuli tui illi<us $>$, sicut liberasti Israelitas de monte Iebu<sa>ei, et Ionam de ventre ceti, et Daniel de lacu leonum. (CP 1465, Fragment d'une messe gallicane inédite conservée par le manuscrit BN lat. 256) (33 $^{33}$

Libère, Seigneur, l'âme de ton serviteur, comme tu as libéré le peuple d'Israël du mont des Jébuséens, et Jonas du ventre de la baleine, et Daniel de la fosse des lions. 
Ventris piscis est attesté au viıre ou au IXe siècle dans le bénédictionnaire de Freising, ainsi qu'au xıı siècle dans le Liber mozarabicus sacramentorum, qui pourrait reprendre un fond liturgique antérieur à la conquête arabe de 712 :

Liberet vos de omnibus malis et peccatis et omni blasphemia, qui liberavit Ionam de ventre piscis, et liberavit Petrum de mari, et Paulum de vinculis.

(Corpus benedictionum, 1468, Bénédictionnaire de Freising)

Qu'il vous libère de tous les maux et péchés et de tout blasphème, celui qui a libéré Jonas du ventre du poisson et a libéré Pierre de la mer et Paul de ses chaînes.

Secundum quam praefigurationem et Iona ventre piscis tribus diebus detentus egreditur

(Liber mozarabicus sacramentorum)

Selon cette préfiguration, Jonas aussi est sorti du ventre du poisson après avoir été détenu pendant trois jours.

La persistance de ventris ceti, dans la liturgie et chez les auteurs médiévaux, s'explique par la traduction de la Vulgate de l'évangile de Matthieu, en 12, 40 :

sicut enim fuit Ionas in uentre ceti tribus diebus et tribus noctibus sic erit Filius hominis in corde terrae tribus diebus et tribus noctibus

De même, en effet, que Jonas fut trois jours et trois nuits dans le ventre de la baleine, ainsi le Fils de l'homme sera trois jours et trois nuits dans le cœur de la terre.

Celle-ci a en effet conservé le ceti vieux-latin. Le texte de l'évangile, sans doute davantage lu, cité et commenté que le livre de Jonas, a contribué à ancrer dans la culture biblique collective la traduction du verset évangélique citant le verset originel et non celle du verset originel.

Deux autres citations bibliques dans des textes liturgiques témoignent du même mécanisme dans lequel la réception d'un verset des livres des Douze Prophètes est contaminée ou supplantée par l'emploi néo-testamentaire de ce verset. 


\subsection{Zacharie 9, 9}

La préface 235 du Corpus praefationum témoigne de la diffusion d'une traduction hiéronymienne qui nest pas celle donnée dans la Vulgate mais celle effectuée par Jérôme sur la Septante dans un de ses commentaires bibliques et transmise par le texte des Évangiles :

Quapropter supplices te, Domine, deprecamur, ut benedicas hos ramos arborum, quos tui famuli in suis suscipientes manibus in occursum tui properare, teque benedicere et glorificare desiderant. Ecce Ierusalem sedens super asinam rex mansuetus advenisti. $(C P, 235)$

C'est pourquoi, en te suppliant, Seigneur, nous te prions de bénir ces rameaux des arbres, que tes serviteurs, en les recevant dans leurs mains, désirent approcher de toi ; ils désirent te bénir et te glorifier. Voici, Jérusalem, que vient ton roi, clément, assis sur une ânesse.

Elle est attestée pour la bénédiction des rameaux et des branches d'olivier dans le Sacramentaire léonien au vie siècle et dans le pontifical romano-germanique du xe siècle. Elle subsiste également dans le Sacramentaire aragonais d'Osca (du xıre siècle) qui était utilisée pour la messe du dimanche des Rameaux ou pour la bénédiction des rameaux et des branches d'olivier. Dans une version plus courte de bénédiction des rameaux, cette prière est attestée à la fin du viıIe siècle dans un sacramentaire gélasien grégorianisé originaire de Regensburg et passé à Prague, et au Ixe siècle dans un sacramentaire originaire d'Essen et dans un fragment de sacramentaire originaire de Padoue et conservé à Salzbourg. Cette pièce cite Zacharie 9, 9, dont la traduction de la Vulgate est la suivante :

exulta satis filia Sion iubila filia Hierusalem et saluator ipse pauper et ascendens super asinam

Exulte grandement, fille de Sion, jubile, fille de Jérusalem, car ton sauveur vient lui-même, pauvre et assis sur une ânesse

La traduction Vulgate de l'évangile de Matthieu $(21,5)$ porte quant à elle ce texte :

dicite filiae Sion ecce rex tuus uenit tibi mansuetus et sedens super asinam 
Dites à la fille de Sion : voici que vient pour toi ton roi, doux et assis sur une ânesse

La préface cite-t-elle le livre de Zacharie ou l'évangile de Matthieu? La présence dans le texte liturgique du nom Hierusalem tend à faire penser que le rédacteur avait le livre de Zacharie en tête même s'il connaissait le verset de Matthieu. Mansuetus et super asinam ne sont pas des marqueurs de leçons des Vieilles Latines pour le livre de Zacharie. Ils se trouvent en revanche dans le Commentaire sur Amos de Jérôme, où celui-ci donne une traduction sur la Septante de ce verset précis du livre de Zacharie :

In Zacharia quoque legimus, quod Euangelii testimonio comprobatur, et refertur ad praesentiam saluatoris: Gaude nimis, filia Sion, praedica, filia Hierusalem : ecce rex tuus uenit tibi iustus et saluator, ipse mansuetus, et ascendens super asinam et pullum asinae (3.6, 1. 303 SL 76)

Nous lisons aussi dans le livre de Zacharie ce qui est démontré par le témoignage de l'Évangile et qui concerne la présence du Sauveur : Réjouis-toi grandement, fille de Sion ; proclame-le, fille de Jérusalem : voici que vient pour toi ton roi, le juste et le sauveur, lui qui est doux, monté sur une ânesse et le petit d'une ânesse.

Dans son Traité sur les Psaumes, Hilaire de Poitiers cite nommément le livre de Zacharie en employant le verset de la traduction du livre de Matthieu dans la Vulgate. Certes, celle-ci diffère de la traduction du verset sur la Septante donné par Jérôme dans son commentaire sur Amos, mais le passage d'Hilaire prouve qu'il était possible de citer le verset de Matthieu en voulant citer Zacharie, et donc que la traduction vieille latine de Zacharie, proche de celle Vulgate de Matthieu, a inspiré des citations ultérieures de Zacharie car elle était ancrée dans les mémoires.

\subsection{Habacuc 2, 4}

Une bénédiction pour l'office des matines de la fête de saint Saturnin de l'Oracional Visigotico (des viI-VIIIe siècles) emploie également un verset d'un des livres des Douze Prophètes, celui d'Habacuc, en le citant dans la version dans laquelle les traductions des épîtres le citent : 
Et qui ex fide sua iustum vivere facit, ex pietate peccatores ab omni solvat contagione delicti. Amen.

Et toi qui fais vivre le juste en raison de sa foi, par ta miséricorde, délivre les pécheurs de toute souillure du péché. Amen.

(Corpus benedictionum, 2044)

Les traductions hiéronymiennes auxquelles il est possible que la préface ait emprunté sont celles des deux livres néo-testamentaires qui citent ce verset d'Habacuc plutôt que le verset d'Habacuc lui-même :

ecce qui incredulus est non erit recta anima eius in semet ipso iustus autem in fide sua vivet $\left(\mathrm{Hab}_{2}, 4\right)$

Celui qui est incrédule n'aura point l'âme droite en lui-même ; mais le juste vivra de sa foi.

quoniam autem in lege nemo iustificatur apud Deum manifestum est quia iustus ex fide vivit ( $\left.\mathrm{Gal}_{3}, 11\right)$

Car il est clair que personne n'est justifié par la loi devant Dieu, puisque 'Le juste vit de la foi'.

iustus autem meus ex fide vivit quod si subtraxerit se non placebit animae meae (He 10, 38)

Or mon juste vivra de la foi ; car s'il se retire, il ne sera pas agréable à mon âme.

À l'exception d'Augustin, dans le Speculum (15), et de Jérôme, dans son Commentaire sur Habacuc $(1,2)$, aucun Père latin ne cite l'un de ces versets avec la formule in fide. Cyprien, dans le De mortalitate (3), emploie fide uiuere, mais les Pères de l'Église - y compris Augustin dans ses autres œuvres - sont presque unanimes pour citer la forme ex fide uiuere. Bien qu'il provienne du livre d'Habacuc, ce sont probablement plus souvent les formes vieilles-latines des citations du verset dans les épîtres néo-testamentaires, conservées dans la révision de la Vulgate, quont en tête les rédacteurs liturgiques qui le citent, et parmi eux le rédacteur de l'oraison ici étudiée. Si on trouve quelques attestations de in fide uiuere au Moyen-Âge, aucune ne se trouve dans un document liturgique. La bénédiction de l'Oracional Visigotico est la seule pièce liturgique de notre corpus à citer ce verset, mais elle témoigne, 
comme les mentions et citations de Jonas 2, 1, d'une persistance de la tradition textuelle vieille-latine par le biais des traductions des livres néotestamentaires la citant.

\section{CONCLUSION}

Si les citations et mentions bibliques tirées des Douze Prophètes ne sont pas un corpus suffisant pour étudier la complexité du rapport aux traductions bibliques des livres liturgiques latins, elles permettent néanmoins de mettre en évidence quelques pistes. Tout d'abord, la pauvreté de la transmission directe de la tradition vieille-latine de ces livres rend nécessaire la comparaison de plusieurs sources et la mise en relation d'hypothèses de datation de ces sources pour établir si un verset est vieux-latin ou non : les Douze Prophètes sont des livres pour lesquels on manque de manuscrits et l'établissement de l'histoire du texte en est fragilisé car nous sommes dépendants de témoins secondaires. Elles témoignent néanmoins de l'interférence fréquente entre texte vieux-latin et texte hiéronymien, pour peu que la révision de la traduction d'un livre du Nouveau Testament reprenne une traduction vieille-latine d'un livre du canon hébraïque. Elles donnent en même temps à lire des exemples de traductions vieilles-latines ayant survécu au travail de Jérôme parce qu'elles étaient ancrées dans la culture biblique commune. Elles incitent enfin à une étude détaillée des sources bibliques des livres liturgiques, recueil par recueil, puisqu'elles mettent en évidence le fait que les rédacteurs d'un même livre liturgique, comme le Missel de Bobbio, ont puisé en même temps à la traduction hiéronymienne et à des traductions antérieures. 


\section{BIBLIOGRAPHIE}

Adriaen, Marc, dir. Jérôme: Commentarii in prophetas minores; In Ioelem. CCSL 76.2 1.290, 298, 342, 379, 425. Brepols : Turnhout, 1969-1970.

Berger, Samuel. Histoire de la Vulgate pendant les premiers siècles du Moyen Âge. Berger-Levrault : Nancy, 1893.

Cahill, Marc, dir. Expositio Euangelii secundum Marcum. CCSL 82. Turnhout : Brepols, 1997.

Canellis, Aline, dir. Jérôme : Préfaces aux livres de la Bible. Paris : Le Cerf, 2017.

De Bruyne, Donatien. «Une messe gallicane inédite Pro defuncto ». Revue Bénédictine 34 (1922) : 156-158.

Dekkers, E., J. G. P. Borleffs, R. Willems, R. F. Refoulé, G. F. Diercks et A. Kroymann., dir. Tertullien : Adversus Marcionem. CCSL 1.4.15. Brepols : Turnhout, 1954.

Dumas, Antoine, dir. Liber Sacramentorum Gellonesis. CCSL 159. Brepols : Turnhout, 1981.

Frede, Hermann Josef, Herbert Stanjek et Uwe Fröhlich. « Reste einer Prophetenhandschrift ». Dans Vetus Latina-Fragmente zum Alten Testament. Herder : Freiburg, 1996.

Gryson, Roger. Altlateinische Handschriften: Manuscrits vieux latins; Répertoire descriptif, première partie : Mss 1-275. Freiburg : Herder, 1999.

Heiming, Odilo, osB, dir. Liber sacramentorum Augustodunensis. CCSL 159в. Turnhout: Brepols, 1984.

Marbach, Carl, dir. Carmina Scripturarum scilicet Antiphonas et Responsoria ex sacro Scripturae fonte in libros liturgicos Sanctae Ecclesiae Romanae derivata. Le Roux : Strasbourg, 1907.

Metzger, Bruce. The Early Versions of the New Testament Their Origin, Transmission and Limitations. Oxford: Clarendon Press, 1977.

Moeller, Eugène, dir. Corpus benedictionum pontificalium. CCSL 162-162C. Turnhout: Brepols, 1971-1979.

- dir. Corpus praefationum. CCsL 161-161D. Turnhout : Brepols, 1980-1981.

— J Jean-Marie Clément et Wallant Coppieters't, dir. Corpus orationum. CCSL 160-160M. Turnhout: Brepols, 1992-2004.

Morin, Germain, dir. Liber Comicus, sive lectionarius missae quo Toletana ecclesia ante annos mille et ducentos utebatur. Analecta Maredsolana 1. Maredsous : Monastère Saint-Benoît, 1893. 
Mundó, Anscari M. « La datación de los códices liturgicos visigóticos toledanos ». Hispania Sacra 18.1 (1965) : 1-25.

Quentin, Henri. Mémoire sur l'établissement du texte de la Vulgate. Rome : Desclée, 1922.

Rose, Els, dir. Missale Gothicum e codice Vaticano Reginensi latino 317 editum. CCSL 159D. Turnhout: Brepols, 1992-2004.

Saint-Roch, Patrick, dir. Liber Sacramentorum Engolismensis. CCSL 159C. Brepols : Turnhout, 1987.

Salmon, Pierre. « Le texte biblique des lectionnaires mérovingiens ». Dans La Biblia nell'alto Medioevo. Spolète : Centro italiano di studi sull'alto Medioevo, 1962.

Steinhauser, Kenneth. « Job in Patristic Commentaries and Theological Works ». Dans A companion to the Job in the Middle Ages, sous la direction de Franklin Harkins et Aaron Canty. Leiden : Brill, 2017.

- Anonymus in Iob commentarius, sous la diréction de Hildegund Müller et Dorothéa Weber. Csel 96. Wien : De Gruyter, 2006.

Westra, Liuwe H. The Apostle's Creed: Origin, History, and Some Early Commentaries. Turnhout : Brepols 2002. 


\section{RÉSUMÉ}

La diffusion des révisions hiéronymiennes des Bibles latines s'est faite notamment à travers les textes liturgiques latins. La présente communication s'intéresse à l'utilisation de l'œuvre du moine de Bethléem dans les prières des livres liturgiques (missels, sacramentaires et bénédictions pontificales). Elle est centrée sur les citations des livres dits "des petits prophètes". Si l'œuvre de Jérôme s'impose progressivement dans la vie liturgique occidentale à partir de la deuxième moitié du vire et du viıre siècle, plusieurs missels et sacramentaires comportent aussi bien des citations de la révision de Jérôme que de versions des Vieilles latines. C'est notamment le cas de sacramentaires irlandais de la fin du viıe siècle et du missel gallican dit Missale Gothicum, datant des années 70o. À cette époque, l'œuvre de Jérôme nest donc pas encore utilisée par la liturgie comme un ensemble unifié. La citation et l'allusion aux anciennes traductions latines persiste même dans la péninsule ibérique et la liturgie mozarabe jusqu'au Xıre siècle. Une étude détaillée des allusions au livre II de Jonas montre que des morceaux de versets issus des Vieilles latines se sont maintenus pendant tout le Moyen-Âge dans des livres ayant recours à la Vulgate, peut-être parce qu'ils étaient passés dans la culture biblique collective. Cet article souligne donc la nécessité d'une analyse détaillée, livre liturgique par livre liturgique, pour étudier les citations de la Bible dans la liturgie latine, car une même pièce liturgique peut emprunter à plusieurs traductions différentes.

MOTS-CLÉS: Bibles latines, Douze Prophètes, livres liturgiques, Vieilles Latines, Jérôme de Stridon 


\section{THE RECEPTION OF JEROME'S REVISION OF BIBLICAL TRANSLATIONS IN THE LATIN LITURGICAL BOOKS (5TH- 12TH CENTURY): THE CASE OF THE TWELVE PROPHETS}

\section{ABSTRACT}

The paper focuses on how the Latin liturgical prayers dealt with the different Latin biblical translations, Old Latin and Jerome's translations and revisions, from the 5 th up to the 12 th century. Many studies on the spreading of Jerome's translations have focused on Latin Bible manuscripts or fragments, the Latin Fathers' quotations of the Bible, and the Latin lectionaries' quotations of the Bible. The present study chooses to survey the liturgical books of prayers, specifically the Twelve Prophets' translations; while the corpus is not a big one, it offers noteworthy results. First presented are quotations or mentions of a Latin verse where translation is identical in the Vetus Latina and the Vulgate. Then cases where Vulgate is used and cases where Old Latin is used are analyzed. It is not always easy to identify the translation used in the liturgical book, and one can sometimes only compare different assumptions on biblical-inspired liturgical texts to know whether its source is Old Latin or Vulgate. Moreover, translations of an Old Testament verse and a New Testament one are sometimes interfering. This happens mainly when the New Testament verse, while quoting the Old Testament one, retains the Old Latin translation, even in the Vulgate version. Samples of verses whose Old Latin survived Jerome's translation are provided. The paper shows how one liturgical book can draw on both Old Latin and Vulgate, even within the same item, and stresses the need for a detailed analysis, liturgical book by liturgical book, to study the quotations from the Bible in the Latin liturgy.

KEYWORDS: Latin Bibles, Twelve Prophets, liturgical books, Vetus Latina, Jerome of Stridon 


\section{ŠIRJENJE HIERONIMOVE REVIZIJE PREVODA SVETEGA PISMA V LATINSKIH LITURGIČNIH KNJIGAH (5.-12. STOLETJE) NA PRIMERU MALIH PREROKOV}

\section{IZVLEČEK}

Širjenje Hieronimovega prevoda je pri latinski Bibliji potekalo zlasti prek latinskih liturgičnih besedil. Razprava se posveča rabi besedil betlehemskega meniha pri molitvah v liturgičnih knjigah (misali, zakramentariji in papeški blagoslovi). Osredotoča se na odlomke iz takoimenovanih »malih prerokov«. Čeprav se je Hieronimovo delo v zahodni liturgiji od druge polovice 7 . in 8. stoletja dalje vse bolj uveljavljalo, je več misalov in zakramentarijev še vedno vključevalo tako odlomke iz Hieronimove revizije kot starejše latinske različice (Vetus Latina). To še posebej velja za irske zakramentarije s konca 7. stoletja in za galikanski misal, znan kot Missale Gothicum, iz 8. stoletja. Hieronimovega dela kot celote $\mathrm{v}$ liturgiji takrat torej še niso uporabljali. Citate in aluzije na stare latinske prevode je bilo najti celo na Iberskem polotoku in v mozarabski liturgiji do 12. stoletja. Podrobna analiza aluzij na drugo poglavje preroka Jona pokaže, kako so se deli verzov starejših latinskih prevodov ohranili skozi srednji vek tudi v knjigah, kjer je bila sicer v rabi Vulgata, morda zato, ker so prešli v kolektivno svetopisemsko kulturo. Članek zato poudarja potrebo po podrobni analizi vseh liturgičnih knjig ter po analizi citatov iz Svetega pisma v latinski liturgiji, saj je pri enem liturgičnem besedilu mogoče najti več različnih prevodov.

KLJUČNE BESEDE: latinske Biblije, dvanajst prerokov, liturgične knjige, Vetus Latina, Hieronim iz Stridona 